\title{
Interference Reduction In Cognitive Networks via Scheduling
}

\author{
Patrick Mitran, Member, IEEE
}

\begin{abstract}
In this letter, we first define a cognitive network to be useful if at least one node can be scheduled to transmit without causing significant simultaneous interference to any primary user and then investigate the interaction between secondary network size and the probability of the secondary network being useful.

First the size of the primary network is fixed, and we analyze how quickly the interference threshold limit of the primary network can be reduced as a function of secondary network size. Here there is a tradeoff between the rate of interference threshold reduction and the probability that the secondary network is useful which is completely characterized for Rician fading.

We then allow both networks to grow simultaneously. Here the tradeoff is determined in the regime that the interference decreases sufficiently fast for Rayleigh fading. We also investigate the effect of primary channel correlation.

Finally, we say that the secondary network is $\ell$-useful provided at least one of any $\ell$ secondary nodes can be scheduled. We show that in the asymptotic regime, the probabilities of the secondary network being $\ell$-useful are uniquely related and do not depend on the asymptotic behavior of the interference threshold, the rates at which the networks grow or even the distribution of the fading.
\end{abstract}

Index Terms - Cognitive radio, interference, scheduling.

\section{INTRODUCTION}

Spurred by the observation that many licensed frequency bands are vacant most of the time, cognitive radio has been proposed as a means to increase overall spectral efficiency. In a cognitive radio based on opportunistic spectrum access (OSA), the cognitive radio, or secondary user, is permitted to transmit in the licensed frequency band of a primary (or incumbent) user so long as the primary user does not itself employ the band. When a primary user resumes transmission, the secondary user must vacate the channel. Perhaps the best known example of this technology is the emerging IEEE 802.22 standard whose aim is to exploit unoccupied TV spectrum. A major challenge in OSA is the identification of spectral holes, called spectrum sensing. Some methods to accomplish this are given in [6], [3], although this list is far from comprehensive.

An alternative approach to OSA is to allow the cognitive radio to transmit simultaneously with the primary. This strategy is often called interference mitigation, and the secondary user must be careful that the interference caused to the primary is not "significant" or "harmful". In [2], [7], [8],

Manuscript received August 14, 2008; revised January 14, 2009; accepted March 25, 2009. The associate editor coordinating the review of this paper and approving it for publication was A. Yener. This work was presented in part at the 24th Biennial Symposium on Communications.

The author is with the Department of Electrical and Computer Engineering, University of Waterloo, Waterloo, Ontario N2L 3G1 (email: pmitran@ecemail.uwaterloo.ca). the interference mitigation approach is taken and transmit power control policies are determined in the case of a primary transmitter/receiver and secondary transmitter/receiver. It has also been noted that from an information theoretic point of view, under some suitable geometric constraints, the secondary can in principle help the primary as it may be able to recover the primary message before the primary has finished transmitting [1].

In this letter, we follow the interference mitigation approach, that is to say, the maximum interference that any secondary user is allowed to generate at any primary receiver is fixed to a specified threshold. We do so in the setting of colocated primary and secondary networks, i.e., the secondary network has the potential to interfere with the primary. While it is evident that many secondary users will not be able to transmit due to interference caused at primary receivers, we say that the cognitive network is useful if at least one secondary user can be found whose transmission would not exceed the interference threshold. Except for section VI, in this letter, only one secondary node transmits at a time and the scheduler is free to select any user whose transmission would be below the interference threshold. In practice, the scheduler is likely to break ties based on the specific demands of its network but in this letter its primary task is to exploit channel fading to find a suitable secondary user for transmission. If no secondary transmission can be below the interference threshold, the secondary network is said to be in outage. Thus, there is a tradeoff between the sizes of the networks, the interference threshold (as a function of the sizes) and the probability of secondary outage. The formulation used here has the advantage that it does not assume any particular behavior or configuration for the primary network.

The contributions of this letter are as follows.

1) We analyze the interference caused to the primary network in the asymptotic of large secondary networks. The tradeoff is characterized exactly for Rician fading with a fixed primary network size and provided the interference threshold decays fast enough, for Rayleigh fading with increasing primary network size.

2) We analyze the effect of primary channel correlation.

3) We quantify the utility of the secondary network by the notion of $\ell$-usefulness which we believe to be novel.

4) We show that for non-vanishing limits to the outage probability, there are no tradeoffs for the system designer.

Finally, we note that scheduling users whose interference is below a threshold is analogous to a more traditional downlink problem. Specifically, in a fading downlink channel, it is 
known that only scheduling the user with the strongest channel gain maximizes throughput. Here, our scheduler exploits channel fading for a different purpose: to minimize interference.

This letter is structured as follows. In section II we present the model that will be employed in this letter and a general tradeoff limit. In section III, we study the asymptotic relationship between the interference threshold, the number of secondary users, and the probability of secondary outage in the case of a fixed size primary network. In section IV, we require that both the primary and secondary networks grow and again analyze the asymptotic relationship. In section $\mathrm{V}$ we look at the effect of primary channel correlation. In section VI we generalize the notion of usefulness to $\ell$-usefulness. Finally, in section VII, we conclude this work.

\section{PRELIMINARIES}

\section{A. Model and Notation}

The size of the primary (resp. secondary) network is denoted by $m$ (resp. $k$ ). The complex channel gain between primary user $j=1, \ldots, m$ and secondary user $i=1, \ldots, k$ is denoted by $h_{i, j}$. Thus the ratio of transmitted to received power is $G_{i, j}=\left|h_{i, j}\right|^{2}$. The $G_{i, j}$ have cumulative distribution function (CDF) $F(x)=P\left[G_{i, j} \leq x\right]$ and in sections II - IV are taken to be i.i.d. This model reflects networks where fading is the dominant effect. We further assume that $F(0)=0$ and $F(x)<$ 1 for $x<\infty$.

We assume that when a secondary transmits, it does so with power $P$, which for simplicity is taken to be $P=1$ and thus the interference caused by secondary $i$ to primary $j$ is $G_{i, j}$.

The effect of the secondary network on the primary is modeled by the interference caused to the primary. The maximum allowable limit is denoted by $I(k)$, an explicit function of secondary network size, and is selected so that the primary system is not adversely affected. When the secondary network cannot operate at or below this threshold, it must stop transmission and is thus said to be in outage. The probability of secondary outage is denoted by $P_{\text {out }}(k)$.

We take an optimistic view and, except for section VI, attempt to schedule at most one secondary user at a time and say that the secondary network is useful if this can be accomplished; otherwise the secondary network is said to be in outage. Alternatively, in a low duty-cycle secondary network, it may be sufficient to be able to schedule one secondary user in one time slot out of every $F$ time slots (e.g., $F=100$ ). All the results below still hold then provided we replace $k$ with $k F$ under the assumption that independent fading is realized in every time slot.

In section III, we fix $m$, and investigate the behavior of $I(k)$ as $k \rightarrow \infty$ in terms of $P_{\text {out }}(k)$ and derive the fundamental tradeoff in (14) for Rician fading provided only that $\lim _{k \rightarrow \infty} I(k)=0$.

In section IV, we allow $m$ to depend on $k$. In particular, we require $\lim _{k \rightarrow \infty} m(k)=\infty$ and analyze the tradeoff between $P_{\text {out }}(k)$ and the rates at which $m(k) \rightarrow \infty$ and $I(k) \rightarrow 0$. In this case,

$$
\lim _{k \rightarrow \infty} \frac{\ln P_{\text {out }}(k)}{k[I(k)]^{m(k)}}=-1,
$$

for Rayleigh fading provided that $\lim _{k \rightarrow \infty} I(k) m(k)=0$.

\section{B. General Tradeoff Limit}

In this section, we provide a general tradeoff limit,

Proposition 1: Provided we have that $\lim _{k \rightarrow \infty}[F(I(k))]^{m(k)}=0$, then,

$$
\lim _{k \rightarrow \infty} \frac{\ln P_{\text {out }}(k)}{k[F(I(k))]^{m(k)}}=-1,
$$

for any cognitive network.

Remark: The condition that $\lim _{k \rightarrow \infty}[F(I(k))]^{m(k)}=0$ is always satisfied if both $I(k)<\infty$ and $\lim _{k \rightarrow \infty} m(k)=\infty$ or if $\lim _{k \rightarrow \infty} I(k)=0$.

Proof: Since the cumulative distribution function of the interference power gain $G_{i, j}$ between secondary user $i$ and primary user $j$ is $F(x)$, the CDF of the worst interference $G_{i, \max }:=\max \left\{G_{i, 1}, G_{i, 2}, \ldots, G_{i, m}\right\}$ that would be generated by secondary $i$ to any primary is given by

$$
F_{G_{i, \max }}=[F(x)]^{m(k)} .
$$

Now, the secondary network is useful if and only if $G=$ $\min \left\{G_{1, \max }, G_{2, \max }, \ldots, G_{k, \max }\right\}$ is less or equal to $I(k)$ since then there is at least one secondary $i$ for which $G_{i, \max } \leq I(k)$. Furthermore, since the CDF of $G$ is given by

$$
\begin{aligned}
F_{G}(x) & =1-\left(1-F_{G_{i, \max }}(x)\right)^{k} \\
& =1-\left(1-[F(x)]^{m(k)}\right)^{k},
\end{aligned}
$$

then the probability of outage is

$$
\begin{aligned}
P_{\text {out }}(k) & =P[G>I(k)] \\
& =\left(1-[F(I(k))]^{m(k)}\right)^{k} .
\end{aligned}
$$

Thus, our problem reduces to studying the behavior of $(1-$ $\left.[F(x)]^{m(k)}\right)^{k}$. However,

$$
\begin{aligned}
\ln P_{\text {out }} & =k \ln \left(1-[F(I(k))]^{m(k)}\right) \\
& =-k\left([F(I(k))]^{m(k)}+O\left([F(I(k))]^{2 m(k)}\right)\right) .
\end{aligned}
$$

Therefore,

$$
\frac{\ln P_{\text {out }}(k)}{k[F(I(k))]^{m(k)}}=-1+O\left([F(I(k))]^{m(k)}\right) .
$$

Finally, since $\lim _{k \rightarrow \infty}[F(I(k))]^{m(k)}=0$, the result follows.

As an immediate corollary of this result, it follows that if $\lim _{k \rightarrow \infty}[F(I(k))]^{m(k)}=0$ then exponential in $k$ behavior of $P_{\text {out }}(k)$ is not possible, while if $[F(I(k))]^{m(k)}$ has a non-zero limit, then from (7), $P_{\text {out }}(k)$ decays exponentially.

\section{FIXED SiZE Primary Network}

In this section, we analyze the behavior of the probability of outage for Rician fading in the case that the primary network size $m(k)$ is kept fixed to $m>0$ while the secondary network is allowed to grow. For Rician fading, the channel power gain $G_{i, j}$ is given by $G_{i, j}=\left|H_{i, j}\right|^{2}$, where $H_{i, j}$ is a complex Gaussian random variable with mean $\mu$, variance per dimension $\sigma^{2} / 2$ and $\mu^{2}+\sigma^{2}=1$. It is common to characterize Rician fading by it's $K$-factor, defined as $K=\mu^{2} / \sigma^{2}$. 
In terms of interference reduction in co-located networks, since $m$ is fixed, we take $\lim _{k \rightarrow \infty} I(k)=0$ to satisfy the condition of Proposition 1. It follows from (2) that

$$
-1=\lim _{k \rightarrow \infty} \frac{\ln P_{\text {out }}(k)}{k[I(k)]^{m}} \cdot \lim _{k \rightarrow \infty}\left(\frac{I(k)}{F(I(k))}\right)^{m}
$$

The probability density function (pdf) of $G_{i, j}$ is then that of a non-central $\chi^{2}$ distribution with 2 degrees of freedom. Such pdfs are given in $[4$, p. 436] and when expressed in terms of its $K$-factor is

$$
f(x)=(K+1) e^{-(K+1) x-K} I_{0}(2 \sqrt{K(K+1) x}),
$$

where $I_{0}($.$) is a modified Bessel function. Hence,$

$$
\lim _{k \rightarrow \infty} \frac{I(k)}{F(I(k))}=\frac{1}{(K+1) e^{-K}},
$$

and thus,

$$
\lim _{k \rightarrow \infty} \frac{\ln P_{\text {out }}(k)}{k[I(k)]^{m}}=-(1+K)^{m} e^{-m K} .
$$

Since $m$ is kept fixed, exponential decay in $P_{\text {out }}(k)$ is possible only if $I(k)$ converges to a limiting interference threshold $I>0$.

Since interference to the primary network should be minimized, it is interesting to consider a fixed secondary outage probability and use the system resources to maximize the rate at which the interference is reduced.

Corollary 2: For any fixed constant $\gamma>0$, the interference caused by the secondary network in the presence of Rician fading has a maximum rate of decay of

$$
I(k)=\frac{\gamma e^{K}}{(1+K) \sqrt[m]{k}}+o\left(\frac{1}{\sqrt[m]{k}}\right)
$$

where the corresponding outage probability is fixed to

$$
P_{\text {out }}=\exp \left(-\gamma^{m}\right) \text {. }
$$

It follows that even for very moderately sized primary networks, slight increases in the parameter $\gamma$ can result in much greater reliability (reduced outage) as $\gamma$ has greater than exponential effect on $P_{\text {out }}$ while $\gamma$ only linearly influences the worst case interference. Figs. 1 and 2 illustrate this effect for $k=1000$, e.g., say 10 secondary nodes with $F=100$. In this case, we see that by exploiting the fading channel, it is possible to provide between $4 \mathrm{~dB}$ and $9 \mathrm{~dB}$ of interference reduction at $P_{\text {out }}=10 \%$.

Alternatively, if the probability of outage is to be decreased polynomially in $k$, i.e., $P_{\text {out }}(k)=k^{-\gamma^{m}}$, for some $\gamma>0$, then the interference threshold behaves as

$$
I(k)=\frac{\gamma e^{K}}{K+1} \sqrt[m]{(\ln k) / k}+o(\sqrt[m]{(\ln k) / k}),
$$

which again shows the sensitivity of the outage probability to the $m$ th power a multiplicative interference scaling constant.

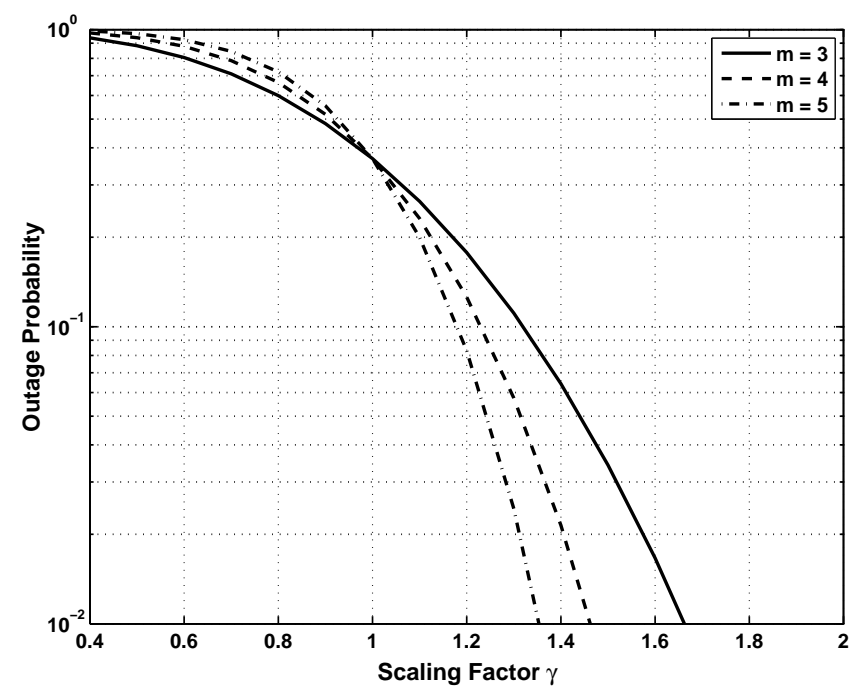

Fig. 1. Outage probability versus scaling factor $\gamma$ in (15) and (16) for $k=1000$ and $K=0$.

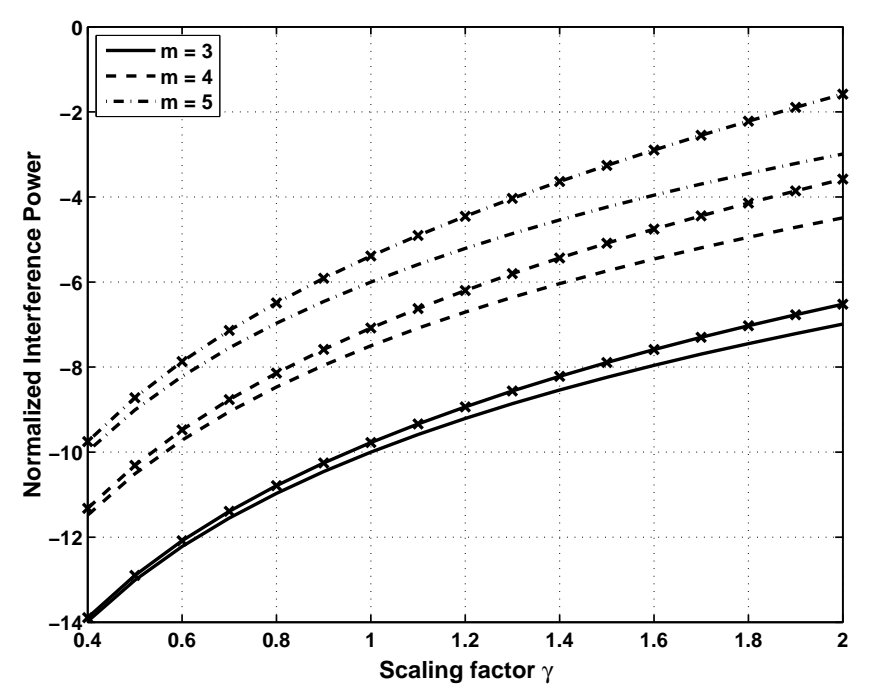

Fig. 2. Interference threshold versus scaling factor $\gamma$ in (15) and (16) for $k=1000$ and $K=0$. Curves without markers are the first term in the approximation in (15) while those with $\times$ are exact.

\section{VARiable Size Primary Network}

We now let the number of primaries $m(k)$ grow with the number of secondary users, $k$, i.e., $\lim _{k \rightarrow \infty} m(k)=\infty$. Since the number of primary users appears in the right hand side of (14), we restrict ourselves to Rayleigh fading $(K=0)$. The main result of this section is that provided that the interference threshold decreases fast enough, a similar limit exists to the fixed primary size case with $K=0$.

Proposition 3: Provided that $I(k)=o(1 / m(k))$ then for Rayleigh fading networks we have

$$
\lim _{k \rightarrow \infty} \frac{\ln P_{\text {out }}(k)}{k[I(k)]^{m(k)}}=-1 .
$$

The difficulty in proving this result is in evaluating the limit of $[I(k) / F(I(k))]^{m(k)}$ in (11) as the exponent is also increasing. 
Proof: By hypothesis, we have that $I(k) \in$ $[-L(m), L(m)]$, where the endpoints of the interval satisfy $L(m)=o(1 / m)$. Furthermore, by Taylor's theorem, we have that in any interval $(-a, a)$,

$$
F(x)=1-e^{-x}=x+R(x),
$$

where $|R(x)| \leq \frac{e^{a}}{2} x^{2}$.

Taking $a=L(m)$, and any sequence $x_{m} \in[-L(m), L(m)]$, $x_{m} \neq 0$, then

$$
\left|\frac{\left(1-e^{-x_{m}}\right)^{m}}{\left(x_{m}\right)^{m}}\right| \leq\left(1+\frac{e^{L(m)}}{2} x_{m}\right)^{m} \rightarrow 1,
$$

as $m \rightarrow \infty$ since $\left|x_{m}\right| \leq L(m)=o(1 / m)$. Similarly,

$$
\left|\frac{\left(1-e^{-x_{m}}\right)^{m}}{x^{m}}\right| \geq\left(1-\frac{e^{L(m)}}{2} x_{m}\right)^{m} \rightarrow 1 .
$$

Therefore, taking $x_{m(k)}=I(k)=o(1 / m(k))$ and $L(m(k))=$ $2 I(k)$, we have that

$$
\begin{aligned}
{[F(I(k))]^{m(k)} } & =\left(1-e^{-I(k)}\right)^{m(k)} \\
& =[I(k)]^{m(k)}+o\left([I(k)]^{m(k)}\right),
\end{aligned}
$$

which proves the result.

\section{EFFect of Channel Correlation}

In this section, we look at the effect of channel correlation on the amount of interference reduction. We model the channel power gain from secondary user $i$ to primary user $j$ by $G_{i, j}=$ $\left|H_{i, j}\right|^{2}$, where $H_{i, j}$ is a zero mean complex Gaussian random variable with unit variance, and for $j \neq j^{\prime}$,

$$
E\left[H_{i, j} H_{i^{\prime}, j^{\prime}}^{*}\right]=\alpha \delta\left(i, i^{\prime}\right),
$$

where $\alpha \geq 0$ and $\delta$ is the delta function. This reflects the scenario where a secondary user sees correlation between the channel gains to the primary users. Equivalently, $H_{i, j}=$ $\bar{H}_{i}+\bar{H}_{i, j}$, where $\bar{H}_{i}$ and $\bar{H}_{i, j}$ are independent circularly symmetric complex Gaussian with variance $\alpha$ and $(1-\alpha)$. We can no longer model $G_{i, \max }$ as having $\operatorname{CDF} F(x)^{m}$ as in (3). Moreover,

$$
\begin{aligned}
& F_{G_{i}, \max }(x) \\
& =P\left[G_{i, 1} \leq x, G_{i, 2} \leq x, \ldots, G_{i, m} \leq x\right] \\
& =E_{\left|\bar{H}_{i}\right|^{2}} P\left[G_{i, 1} \leq x, G_{i, 2} \leq x, \ldots, G_{i, m} \leq\left. x|| \bar{H}_{i}\right|^{2}\right] \\
& =E_{\left|\bar{H}_{i}\right|^{2}} P\left[G_{i, 1} \leq\left. x|| \bar{H}_{i}\right|^{2}\right]^{m},
\end{aligned}
$$

where $\left|\bar{H}_{i}\right|^{2}$ is exponential with mean $\alpha$ and, conditioned on $\left|\bar{H}_{i}\right|^{2}=\left|\bar{h}_{i}\right|^{2}, G_{i, j}, j=1, \ldots, m$, are independent and noncentral $\chi^{2}[4$, p. 436], with pdf

$$
\begin{aligned}
f\left(\left.x|| \bar{h}_{i}\right|^{2}\right)= & \frac{1}{1-\alpha} \exp \left(-\left(\frac{x+\left|\bar{h}_{i}\right|^{2}}{1-\alpha}\right)\right) \\
& \times I_{0}\left(\sqrt{4\left|\bar{h}_{i}\right|^{2} x} /(1-\alpha)\right) .
\end{aligned}
$$

Then, as in Proposition 1,

$$
\lim _{k \rightarrow \infty} \frac{\ln P_{\text {out }}(k)}{k F_{G_{i}, \max }(I(k))}=-1,
$$

where $F_{G_{i}, \max }(x)$ is given by (26) and (27).
While analytic evaluation of $I(k)$ versus $P_{\text {out }}(k)$ is difficult, even in the asymptotic regime, we can evaluate the above numerically as illustrated in Fig. 3 where $P_{\text {out }}$ is fixed at $10 \%$. The figure suggests that as $\alpha$ increases, the amount of interference reduction improves. This is intuitive since as $\alpha$ increases, the primary channels correlate and the secondary users eventually only see a "single" primary channel (i.e., $m=1$ case).

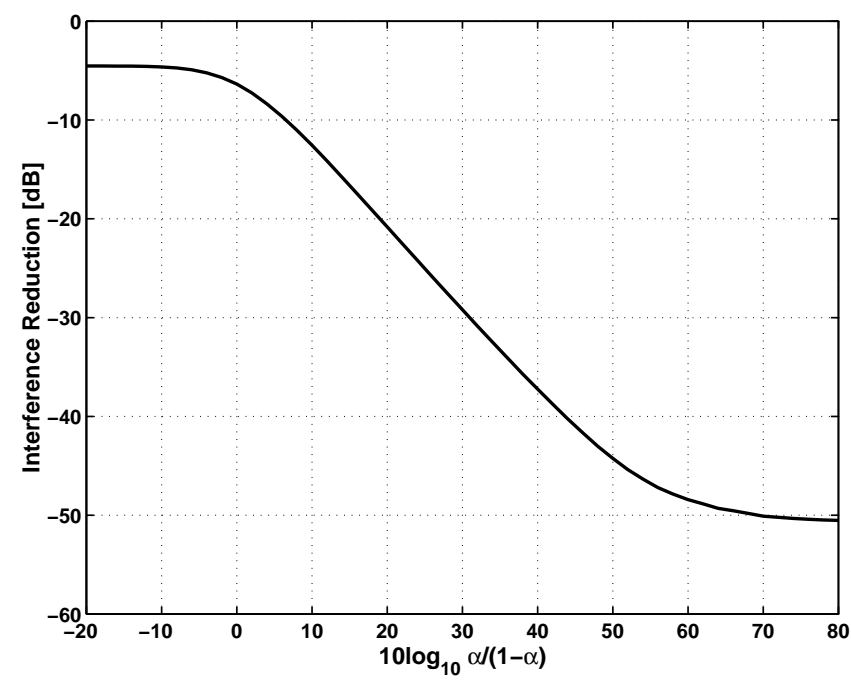

Fig. 3. Interference reduction for $P_{\text {out }}=0.1$ versus correlation of channel gains for $m=5$ and $k=1000$.

\section{VI. $\ell$-USEFUL SECONDARY NETWORK}

We now quantify how large the pool of secondary nodes whose interference would be below the threshold $I(k)$ is. We say that the secondary network is $\ell$-useful if there is a set of $\ell$ nodes, any one of which can transmit (although not necessarily simultaneously) while remaining below the interference threshold $I(k)$. This provides more freedom in terms of which nodes may be scheduled and if $\ell_{1}>\ell_{2}$ then an $\ell_{1}$-useful network provides more utility than an $\ell_{2}$ useful network. For each $\ell$, we can then define $P_{\text {out }}^{(\ell)}$ to be the corresponding outage probability, i.e., the probability that the secondary network is not $\ell$-useful.

The main result of this section is the surprising conclusion that if $P_{\text {out }}^{(\bar{\ell})}(k)$ has a non-trivial limit $0<a<1$ for some $\bar{\ell}$, then $P_{\text {out }}^{(\ell)}(k)$ has a non-trivial limit for all $\ell$ that can be computed as a function $a, \ell$ and $\bar{\ell}$ only and does not depend on the $\mathrm{CDF}$ of the channel power gain, the correlation between the primary channels and a secondary user, the asymptotic behavior $I(k)$ or the asymptotic behavior of $m(k)$. Thus, from a network perspective, there is a unique relationship between the limiting outage probabilities for the notions of $\ell$-useful networks and no room for the system designer to change this tradeoff. This result essentially follows from the Poisson limit. First, we need the following definition.

Definition 4: We define the $\ell$ th order outage function $\xi_{\ell}$ : 
$[0, \infty) \rightarrow(0,1]$ by

$$
\xi_{\ell}(\tau)=e^{-\tau} \sum_{j=0}^{\ell} \tau^{j} / j !,
$$

with the convention that $\xi_{0}(\tau)=e^{-\tau}$ and $\xi_{\ell}(0)=1$.

It is easily verified that the $\xi_{\ell}$ are bijective. The following limit result from [5] (Lemma 1.3.1) will be key.

Lemma 5: Let $\ell>0$ be a fixed integer, $\tau>0$, and $\left\{p_{k}\right\}$ a sequence with $0<p_{n}<1$. Then

$$
\lim _{k \rightarrow \infty} \sum_{j=0}^{\ell}\left(\begin{array}{l}
k \\
j
\end{array}\right) p_{k}^{j}\left(1-p_{k}\right)^{k-j}=\xi_{\ell}(\tau)
$$

if and only if $\lim _{k \rightarrow \infty} k p_{k}=\tau$. Hence, if (30) holds for any one $\ell$, it holds for all such $\ell$.

The key aspect of this result is the only if part: if it holds for any one $\ell$, then it holds for any $\ell>0$, which implies convergence to known quantities for the other values of $\ell$ since the $\xi_{\ell}$ are bijective. Our main result in this section is as follows.

Theorem 6: Suppose that the $I(k)$ and $m(k)$ are chosen such that for some choice of $\bar{\ell}, \lim _{k \rightarrow \infty} P_{\text {out }}^{(\bar{\ell})}(k)=a, 0<$ $a<1$. Then, all the $P_{\text {out }}^{(\ell)}$ have limits in the interval $(0,1)$ and are uniquely determined by $\bar{\ell}$ and the limit $a$.

Proof: First, we observe that the probability that the secondary network is not $\ell$-useful is

$$
\begin{aligned}
P_{\text {out }}^{(\ell)} & =\left(1-F_{G_{1}, \max }(I(k))\right)^{k}+ \\
& +\left(\begin{array}{c}
k \\
1
\end{array}\right) F(I(k))^{m}\left(1-F_{G_{1}, \max }(I(k))\right)^{k-1}+\cdots \\
& \left.+\left(\begin{array}{c}
k \\
\ell-1
\end{array}\right) F_{G_{1}, \max }(I(k))\right)^{(\ell-1)}\left(1-F_{G_{1}, \max }(I(k))\right)^{k-\ell+1} .
\end{aligned}
$$

However, this is exactly the form in Lemma 5 provided we identify $p_{k}$ with $F_{G_{1}, \max }(I(k))$. Therefore, we have that $\lim _{k \rightarrow \infty} P_{\text {out }}^{(\bar{\ell})}(k)=\xi_{\bar{\ell}-1}(\tau)=a$. Since $0<a<1, \tau$ is uniquely determined by $a$ to be $\tau=\xi_{\bar{\ell}-1}^{-1}(a)$. Hence, by Lemma 5 , for any integer $\ell>0$, we then have that $\lim _{k \rightarrow \infty} P_{\text {out }}^{(\ell)}(k)=\xi_{\ell-1}(\tau)$, i.e., the $\ell$-useful outage probabilities are determined uniquely by $\bar{\ell}$ and the limit $a$ and do not depend explicitly on $I(k)$ or $m(k)$.

Finally, it remains to determine the physical interpretation of the parameter $\tau$. First, we introduce the notion of expected strict usefulness of a cognitive network.

We say that a network is strictly $\ell$-useful if it is $\ell$-useful, but not $(\ell+1)$-useful. Then, since the probability that the secondary network is strictly $\ell$-useful is given by $P_{\text {out }}^{\ell+1}(k)-$ $P_{\text {out }}^{\ell}(k)$, the expected strict usefulness of a secondary network is

$$
\mathcal{L}=\sum_{\ell=0}^{\infty} \ell\left(P_{\text {out }}^{(\ell+1)}(k)-P_{\text {out }}^{(\ell)}(k)\right) .
$$

Taking the limit as $k \rightarrow \infty$, then it is easy to verify from Fatou's inequality and intuitive due to the Poisson limit, that the asymptotic expected usefulness is $\tau$.

The fundamental tradeoff between the probability that the network is strictly $\ell$-useful for $\ell=1,2, \ldots, 5$ is illustrated in

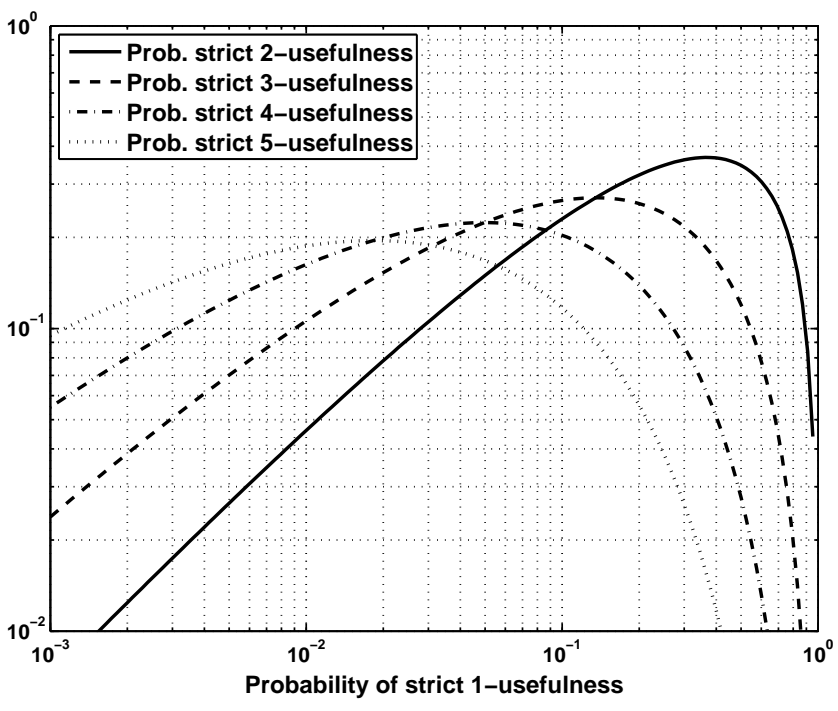

Fig. 4. Tradeoff between limiting probabilities of strict $\ell$-usefulness for $\ell=1, \ldots 5$.

Fig. 4. For example, in the limit of large $k$, to maximize the probability that the network is strictly 5 -useful, the probability of strict 1-usefulness must be approximately 0.02 , regardless of other network parameters.

\section{CONCLUSION}

We defined a secondary network to be useful if at least one node could transmit while maintaining its interference below a given threshold. The limiting behavior between the threshold, outage probability and number of secondary users was characterized. We conclude that for moderate to large secondary networks with low duty cycle, simultaneous transmission that exploits fading is feasible.

In the second part, we extended the notions of usefulness and secondary outage to $\ell$-usefulness. and order $\ell$ outage. Here, it was shown that if the limiting outage probabilities are non-vanishing, then there is only one tradeoff between the limiting probabilities independent of the asymptotic behaviors of network size, interference threshold or even fading models.

\section{REFERENCES}

[1] N. Devroye, P. Mitran, and V. Tarokh, "Achievable rates in cognitive radio channels," IEEE Trans. Inform. Theory, vol. 52, no. 5, pp. 1183-1827, May 2006.

[2] A. Ghasemi and E. S. Sousa, "Fundamental limits of spectrum-sharing in fading environments," IEEE Trans. Wireless Commun., vol. 6, no. 2, pp. 649-658, Feb. 2007.

[3] M. Ghozzi, F. Marx, M. Dohler, and J. Palicot, "Cyclostatilonarity-based test for detection of vacant frequency bands," in Cognitive Radio Oriented Wireless Networks and Communication (CROWNCOM), Mykonos Island, Greece, June 2007.

[4] N. Johnson, S. Kotz, and N. Balakrishnan, Continuous Univariate Distributions. New York:Wiley, 1994, vol. 2.

[5] M. R. Leadbetter, Studies in Probability Theory. MAA, 1978, ch. Extreme value theory under weak mixing conditions, pp. 46-110.

[6] S. M. Mishra, S. ten Brink, R. Mahadevappa, and R. W. Brodersen, "Cognitive technology for ultra-wideband/WiMax coexistence," in Proc. IEEE DySPAN, Dublin, Ireland, 2007.

[7] L. Musavian and S. Aïssa, "Ergodic and outage capacities of spectrumsharing systems in fading channels," in Proc. IEEE Global Telecommun. Conf., Washington, DC, Nov. 2007. 
[8] _ - "Fundamental capacity limits of spectrum-sharing channels with imperfect feedback," in Proc. IEEE Global Telecommun. Conf., Washington, DC, Nov. 2007. 